\title{
Research on the Influence of the Approximated Principal Distance on the Accuracy of Orthophotoplans Out of Historic Polychrome (on Flat and Quasi-Flat Surface)**
}

\section{Introduction}

Photographs used in photogrammetry must be taken with cameras with fixed and stable interior orientation elements (IO elements). To reconstruct a bundle of rays the exact principal distance the moment of taking the picture needs to be known. This requirement is fulfilled by every close-range and aerial photogrammetric camera. However nowadays in close-range photogrammetry professional photogrammetric cameras are used quite rarely. Far more popular are digital SLR cameras. The stability of the IO elements is ensured by turning off the autofocus mode and the mechanical blocking of the lens. It sets limitations for users because after releasing the blocking and changing focus the same IO elements cannot be retrieved, and thus the calibration made for this position of lens loses its value entirely.

In this paper the issue of making orthophotoplans of heritage objects is discussed. Materials made for conservators and public display require the highest possible visual quality and thus geometrical accuracy is not a priority. For a survey the professional photographic equipment is being used, mostly full-frame cameras with prime lenses. The character of objects requires a high resolution of resultant materials with object pixels size $1-0.3 \mathrm{~mm}$.

While working on a site one can come across numerous problems, such as: instability of ground, a very short time one can spend working, impossibility to assure an equal distance from objects while taking the pictures. This may lead to focus (sharpness) loss on some frames. There is a possibility of taking pictures separately focused in the technology of on-the-job calibration [6, 11]. However this technology needs a number of points stabilized on the object and significant depth of field of view, both of which are impossible to achieve on a flat, valuable surface of an historic object. Due to the fact that the highest possible sharpness, provided by the

* AGH University of Science and Technology, Krakow, Poland

** This work was realized within the chair's research project no. 11.11.150.949 
variability of principal distance, and the stability of IO elements are conditions which mutually exclude themselves, the question arises: what errors on orthophotoplans one can expect while using approximated IO elements for the series of photographs?

\section{Variability of Interior Orientation Elements}

Camera calibration is a process in which all IO elements are obtained. It means: principal point, principal distance, radial and decentering distortion. Camera calibration has been already greatly covered by many researches and publications $[3,4$, $6,7,8,11,14]$. Stability of IO elements [12], principal point shift [5, 13], variability of distortion with changing zoom [10] and variability of distortion with changing focus $[4,9]$ have been widely discussed.

Theoretical variability of radial distortion due to a change of focus was presented by D.C. Brown in 1971 [4]. He states that having two calibrations for separate object distances, distortion function for any other can be obtained. Distortion function is defined by formula:

$$
\delta r=K_{1} r^{3}+K_{2} r^{5}+K_{1} r^{7}+\ldots
$$

where:

$$
\begin{aligned}
& \delta r- \text { radial shift caused by radial distortion, } \\
& K_{1^{\prime}}, K_{2^{\prime}}, K_{3^{\prime}} \ldots-\text { radial distortion coefficients, } \\
& r-\text { radial distance. }
\end{aligned}
$$

In the case where one of the distortion functions corresponds to focus at infinity variability of distortion can be presented by formulas:

$$
\begin{gathered}
\alpha_{s}=\frac{s_{1}-f}{s-f} \\
\delta r_{s}=\alpha_{s} \delta r_{s 1}+\left(1-\alpha_{s}\right) \delta r_{\infty s}
\end{gathered}
$$

where:

$$
\begin{aligned}
& \alpha_{s} \text { - variability of radial distortion coefficient, } \\
& f \text { - focal length, } \\
& s_{1^{\prime}} s \text { - distance of object plane, } \\
& \delta r_{s}-\text { distortion function for focus on an object plane at distance } s, \\
& \delta r_{s 1}-\text { distortion function for focus on an object plane at distance } s_{1^{\prime}} \\
& \delta r_{\infty}-\text { distortion function for infinity focus. }
\end{aligned}
$$

Variability of decentering distortion due to change of focus was explained by J.G. Fryer and D.C. Brown [9]. 
Shift of image point coordinates was defined by two equations:

$$
\begin{aligned}
& \Delta x_{s}=\left(1-\frac{f}{s}\right)\left[P_{1}\left(r^{2}+2 x^{2}\right)+2 P_{2} x y\right] \\
& \Delta y_{s}=\left(1-\frac{f}{s}\right)\left[P_{2}\left(r^{2}+2 x^{2}\right)+2 P_{1} x y\right]
\end{aligned}
$$

where:

$$
\begin{aligned}
P_{1}, P_{2} & - \text { decentering distortion coefficients for focus at infinity, } \\
f & - \text { focal length, } \\
s & - \text { distance of object plane, } \\
x, y & - \text { image coordinates, } \\
\Delta x_{s^{\prime}}, \Delta y_{s}- & \text { shift of image coordinates due to decentering distortion for focus } \\
& \text { on object plane at distance } s .
\end{aligned}
$$

For the purpose of this article formulas for shift of image coordinates due to decentering distortion for focus on the object plane at a distance $s$ and $s$ were written and then subtracted, and as the result the formulas (6) and (7) for variability of distortion was obtained:

$$
\begin{aligned}
& \Delta x_{s}-\Delta x_{s^{\prime}}=\left(\frac{f}{s^{\prime}}-\frac{f}{s}\right)\left[P_{1}\left(r^{2}+2 x^{2}\right)+2 P_{2} x y\right] \\
& \Delta y_{s}-\Delta y_{s^{\prime}}=\left(\frac{f}{s^{\prime}}-\frac{f}{s}\right)\left[P_{2}\left(r^{2}+2 x^{2}\right)+2 P_{1} x y\right]
\end{aligned}
$$

S.S. Al-Ajlouni and C. S. Fraser [1] stated, that variability of distortion diminishes significantly beyond focused distances of around 15 times the nominal focal length. Distortion variability due to distance difference in one frame - different than focus plane distance - has to be taken into account. But as Brauer-Burchardt et al. [2] stated, it is significant only if focus distance is 30 times or less of the nominal focal length.

Shift of principal point concerns mostly zoom type lenses. It occurs due to changes of position of the optical lens axis [5]. In the case of prime lenses the principal point shift is linear and proportional to change in the principal distance.

\section{Influence of Approximated Principal Distance on Orthorectification}

In this article, the errors that occur due to the use of the approximated principal distance in the creation of orthophotoplans, are discussed. The approximated principal distance is used in both procedures: exterior orientation elements determination and generation of orthoimages. The formula describing the shift in coordinates was derived on the basis of geometry. 
An assumption was made that for a sample of points bundle of rays will be adjusted correctly regardless of the adopted principal distance and as a consequence the false exterior orientation elements will be calculated. Using those elements to create orthoimages, errors will occur only when points will deviate from plane to which a bundle of rays was adjusted. Such a situation is shown in figure 1.

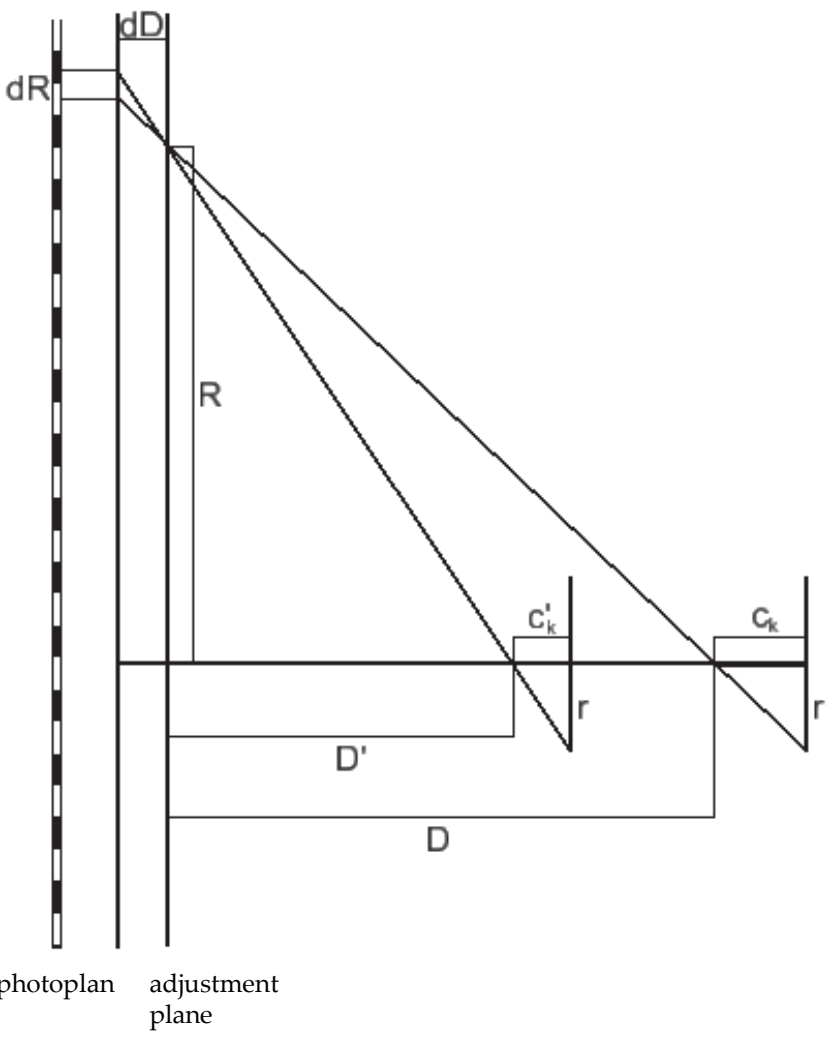

Fig. 1. An adjustment of a bundle of rays using correct and incorrect principal distance together with reconstruction of rays during the process of orthorectification

Adopted designations:

$D$ - real distance from object plane,

$c_{k}$ - real principal distance,

$c_{k}^{\prime}$ - approximated principal distance,

$r$ - maximum radial distance in the picture,

$d D$ - maximum deviation from plane,

$d R$ - shift of point in orthoimage due to approximated $c_{k^{\prime}}$

$D^{\prime}$ - distance from object plane, calculated while using the approximated $c_{k^{\prime}}$

$m_{z}$ - image scale denominator,

$R$ - radial distance on object. 
Regardless of which principal distance will be used in calculating exterior orientation elements, image scale will remain constant. Due to that, the real distance $D^{\prime}$ can be derived from equation:

$$
\frac{D}{c_{k}}=m_{z}=\frac{D^{\prime}}{c_{k}^{\prime}} \Rightarrow D^{\prime}=\frac{D}{c_{k}} \cdot c_{k}^{\prime}
$$

Then using similar triangles, $R$ can be derived from equation:

$$
\frac{r}{c_{k}}=\frac{R}{D+d D} \Rightarrow R=\frac{r \cdot(D+d D)}{c_{k}}
$$

Applying further simple geometric principles, formula (10) describing radial shift of points in resultant orthoimage is obtained:

$$
\begin{gathered}
\frac{r}{c_{k}^{\prime}}=\frac{R+d R}{D^{\prime}+d D} \Rightarrow d R=\frac{r}{c_{k}^{\prime}}\left(D^{\prime}+d D\right)-R \Rightarrow \\
\Rightarrow d R=\frac{r}{c_{k}^{\prime}}\left(\frac{D}{c_{k}} \cdot c_{k}^{\prime}+d D\right)-\frac{r \cdot(D+d D)}{c_{k}} \\
d R=r\left(\frac{D}{c_{k}}+\frac{d D}{c_{k}^{\prime}}\right)-r\left(\frac{D}{c_{k}}+\frac{d D}{c_{k}}\right) \\
d R=r\left(\frac{d D}{c_{k}^{\prime}}-\frac{d D}{c_{k}}\right)
\end{gathered}
$$

\section{Established Conditions of Analytical Research}

Heritage documentation deals with numbers of different objects. Depending on physical properties adequate type of documentation is being made. Orthophotoplans are made only for flat and quasi flat surfaces, like polychrome and stone walls.

The error analysis was performed on how adopting the same interior orientation elements for series of images taken in the autofocus mode will influence the resultant orthophotoplans.

Few assumptions based on typical conditions were made. Three lenses were analyzed, wide angle $-20 \mathrm{~mm}$, normal $-50 \mathrm{~mm}$ and long focus $-150 \mathrm{~mm}$ for full frame camera $-24 \times 36 \mathrm{~mm}$ with pixel size $6 \mu \mathrm{m}$. Two types of surfaces were taken into account, one - polychrome on a wall with deviations from plane $\pm 50 \mathrm{~mm}$ within a frame, second - stone wall with deviations from plane $\pm 150 \mathrm{~mm}$ within a frame. Output pixel size in typical heritage orthophotoplans is $1 \mathrm{~mm}$, but since some objects have small details, pixels can reach up to $0.3 \mathrm{~mm}$. The scale of adjoining photographs should not vary by more than $\pm 10 \%$. 
Errors on orthophotoplans are caused not only by the use of the approximated IO elements but also by type of digital surface models and others. Therefore, it was decided that the error caused by variability of principal distance should not exceed 0.25 pix.

In summary:

- lenses (f): $20 \mathrm{~mm}, 50 \mathrm{~mm}, 150 \mathrm{~mm}$,

- full-frame: $24 \times 36 \mathrm{~mm}$ with pixel size $6 \mu \mathrm{m}$,

- deviations from plane: $\pm 50 \mathrm{~mm}, \pm 150 \mathrm{~mm}$,

- object pixels: $0.3,1 \mathrm{~mm}$

- scale variability $(\Delta s): \pm 10 \%$,

- maximum error: 0.25 pix.

\section{Analysis and Results}

On the basis of the lens equation (11) and established parameters (section 4), the distances from object planes and principal distances were calculated (Tab. 1). Those values were used throughout the analysis:

$$
\frac{1}{f}=\frac{1}{D}+\frac{1}{c_{k}}
$$

where:

$f$ - focal length,

$D$ - real distance from object plane,

$c_{k}$ - real principal distance.

Table 1. Summary of distances from the object plane and principal distances with given parameters

\begin{tabular}{||c|c|c|c|c|c|c|c||}
\hline \multirow{2}{*}{$\begin{array}{c}\text { Focal } \\
\text { length }\end{array}$} & $\begin{array}{c}\text { Real object } \\
\text { distance }\end{array}$ & \multicolumn{3}{|c|}{ Object pixel 1 mm } & \multicolumn{3}{c||}{ Object pixel 0.3 mm } \\
\cline { 2 - 8 } & $\begin{array}{c}\text { Real principal } \\
\text { distance }\end{array}$ & minimum & average & maximum & minimum & average & maximum \\
\hline \hline \multirow{2}{*}{$20 \mathrm{~mm}$} & $D[\mathrm{~m}]$ & 3.000 & 3.333 & 3.667 & 0.900 & 1.000 & 1.100 \\
\cline { 2 - 8 } & $c_{k}[\mathrm{~mm}]$ & 20.134 & 20.121 & 20.110 & 20.455 & 20.408 & 20.370 \\
\hline \multirow{2}{*}{$50 \mathrm{~mm}$} & $D[\mathrm{~m}]$ & 7.500 & 8.333 & 9.167 & 2.250 & 2.500 & 2.750 \\
\cline { 2 - 8 } & $c_{k}[\mathrm{~mm}]$ & 50.336 & 50.302 & 50.274 & 51.136 & 51.020 & 50.926 \\
\hline \multirow{2}{*}{$150 \mathrm{~mm}$} & $D[\mathrm{~m}]$ & 22.500 & 25.000 & 27.500 & 6.750 & 7.500 & 8.250 \\
\cline { 2 - 8 } & $c_{k}[\mathrm{~mm}]$ & 151.007 & 150.905 & 150.823 & 153.409 & 153.061 & 152.778 \\
\hline
\end{tabular}


According to previously shown publications [1,2] variability of distortion is less relevant when the distance from the object plane is greater than 30 times the focal length. For considered lenses that is respectively $0.6 \mathrm{~m}, 1.5 \mathrm{~m}$ and $4.5 \mathrm{~m}$. In this case variability of distortion should not be problematic. However, the difference between distortion function for maximum, minimum and average principal distances were calculated using formulas (6) and (7) shown before:

$$
\alpha_{\min }=\left(\frac{f}{s_{s} r}-\frac{f}{s_{\min }}\right), \quad \alpha_{\max }=\left(\frac{f}{s_{s r}}-\frac{f}{s_{\max }}\right)
$$

Coefficient $\alpha$ - described by formula (12) - shows the degree of difference in decentering distortion between the lens focused on extreme established distant object plane and average. Regardless of a focal length coefficient $\alpha$ is at a level \pm 0.001 for object pixel $1 \mathrm{~mm}$ and \pm 0.002 for object pixel $0.3 \mathrm{~mm}$.

The radial distortion contribution of two distortion functions were calculated using formula (2). For both analysed object pixel percentage of distortion function for lens focused at infinity was about 10, and the percentage of distortion function focused at object plane placed at an average distance was about 90 .

The variability of both distortions radial and decentering with presented values in tables 2 and 3, can be recognized as negligible. Thus assuming one distortion function for a series of photos taken in autofocus mode should not cause significant errors on the resultant orthophotoplan. Also, due to small changes in the principal distance, the principal point shift should not be significant either.

The main problems are radial shifts caused by the approximation of principal distances while counting exterior orientation elements and then performing orthorectification. Using the derived formula (10), the radial shifts, calculated for the case of maximum image radial distance and established conditions, are presented in tables 2 and 3.

Table 2. Summary of extreme, theoretical radial shifts in resultant orthophotoplans due to the approximated principal distance - object pixel $0.3 \mathrm{~mm}$

\begin{tabular}{||c|c|c|c|c|c|c|c|c|c|c||}
\cline { 2 - 11 } \multicolumn{2}{c|}{} & \multicolumn{3}{c|}{ Focal length $20 \mathrm{~mm}$} & \multicolumn{3}{c|}{ Focal length $50 \mathrm{~mm}$} & \multicolumn{3}{c|}{ Focal length $150 \mathrm{~mm}$} \\
\hline \hline \multirow{3}{*}{$\begin{array}{c}\text { Polych. } \\
\text { wall }\end{array}$} & $\Delta s[\%]$ & -10 & 0 & 10 & -10 & 0 & 10 & -10 & 0 & 10 \\
\cline { 2 - 12 } & $d R[\mathrm{~mm}]$ & 0.120 & 0.000 & -0.098 & 0.048 & 0.000 & -0.039 & 0.016 & 0.000 & -0.013 \\
\cline { 2 - 12 } & $d R / O P[\%]$ & 40.06 & 0.00 & -32.78 & 16.02 & 0.00 & -13.11 & 5.34 & 0.00 & -4.37 \\
\hline \multirow{3}{*}{$\begin{array}{c}\text { Stone } \\
\text { wall }\end{array}$} & $\Delta s[\%]$ & -10 & 0 & 10 & -10 & 0 & 10 & -10 & 0 & 10 \\
\cline { 2 - 12 } & $d R[\mathrm{~mm}]$ & 0.360 & 0.000 & -0.295 & 0.144 & 0.000 & -0.118 & 0.048 & 0.000 & -0.039 \\
\cline { 2 - 11 } & $d R / O P[\%]$ & 120.19 & 0.00 & -98.33 & 48.07 & 0.00 & -39.33 & 16.02 & 0.00 & -13.11 \\
\hline \hline
\end{tabular}


Table 3. Summary of extreme, theoretical radial shifts in resultant orthophotoplans due to approximated principal distance - object pixel $1 \mathrm{~mm}$

\begin{tabular}{||c|c|c|c|c|c|c|c|c|c|c||}
\cline { 2 - 11 } \multicolumn{2}{c|}{} & \multicolumn{3}{c|}{ Focal length $20 \mathrm{~mm}$} & \multicolumn{3}{c|}{ Focal length $50 \mathrm{~mm}$} & \multicolumn{3}{c||}{ Focal length $150 \mathrm{~mm}$} \\
\hline \hline \multirow{4}{*}{$\begin{array}{c}\text { Polych. } \\
\text { wall }\end{array}$} & $\Delta s[\%]$ & -10 & 0 & 10 & -10 & 0 & 10 & -10 & 0 & 10 \\
\cline { 2 - 12 } & $d R[\mathrm{~mm}]$ & 0.036 & 0.000 & -0.030 & 0.014 & 0.000 & -0.012 & 0.005 & 0.000 & -0.004 \\
\cline { 2 - 12 } & $d R / O P[\%]$ & 3.61 & 0.00 & -2.95 & 1.44 & 0.00 & -1.18 & 0.48 & 0.00 & -0.39 \\
\hline \multirow{3}{*}{$\begin{array}{c}\text { Stone } \\
\text { wall }\end{array}$} & $d R[\mathrm{~mm}]$ & 0.108 & 0.000 & -0.088 & 0.043 & 0.000 & -0.035 & 0.014 & 0.000 & -0.012 \\
\cline { 2 - 11 } & $d R / O P[\%]$ & 10.82 & 0.00 & -8.85 & 4.33 & 0.00 & -3.54 & 1.44 & 0.00 & -1.18 \\
\hline
\end{tabular}

In tables 2 and $3::$

$d R$ - shift of point in orthoimage due to approximated principal distance,

$\Delta s-$ scale variability of adjoining photographs,

$O P$ - object pixel.

Figures 2 and 3 show visually the results of the conducted studies. Dash lines show the error limits.

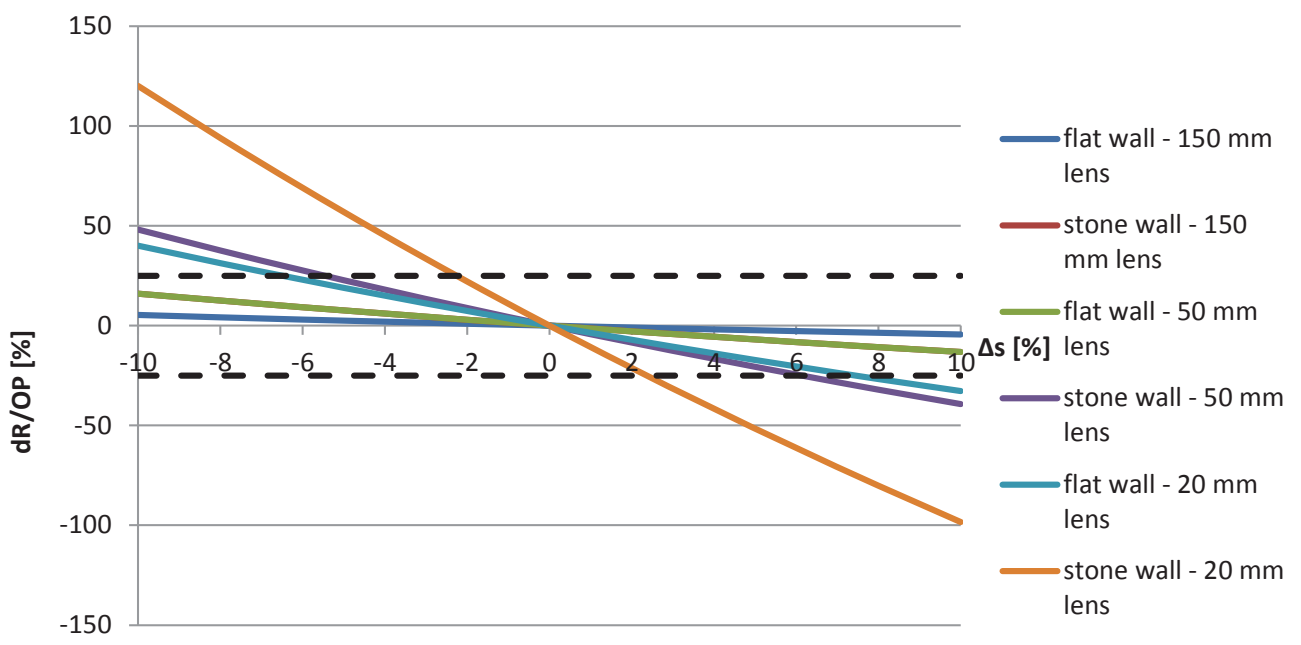

Fig. 2. Graph showing radial shifts on orthophotoplans depending on the variability of the scale - object pixel $0.3 \mathrm{~mm}$ 


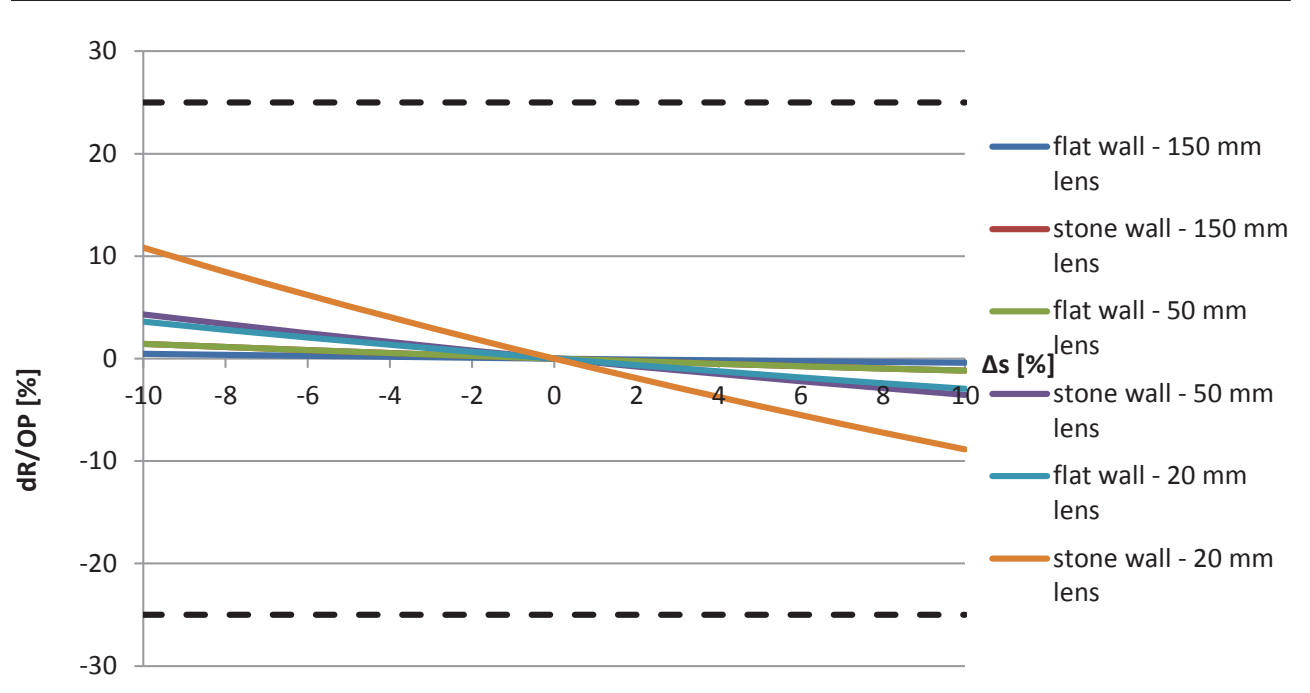

Fig. 3. Graph showing radial shifts on orthophotoplans depending on the variability of the scale - object pixel $1 \mathrm{~mm}$

As the graphs (Figs 2, 3) show, the radial shifts exceed acceptable values only for orthophotoplans with object pixel $0.3 \mathrm{~mm}$ and not for all lenses and surfaces. Though none of the errors for object pixel $1 \mathrm{~mm}$ exceed acceptable values, it would be hard to maintain a scale difference in between pictures in $\pm 10 \%$ brackets for a wide angle lens. For object pixel $0.3 \mathrm{~mm}$ it is safe to say only a long-focus lens is acceptable, but values were calculated for worst case scenarios and it is not common to make orthophotoplans with resolution $0.3 \mathrm{~mm}$ for stone walls, so normal lens should suffice too.

\section{Summary and Conclusions}

This article deliberates the influence of approximated principal distances and other internal orientation elements on the creation of orthophotoplans. A multi-variant theoretical analysis was conducted covering influence depending on the type of lens and resultant resolution.

Results showed quite surprisingly low influence of the use of the approximated IO elements on the creation of orthophotoplans. Errors when using long-focus lens are really insignificant, normal lens will have problems with high resolution orthophotoplans of stone walls, which in practice are never made, and wide angle one should be be used in high resolution jobs both due to error factor and the small variability of distance to object plane.

The technology of using photographs taken in autofocus mode could be successfully implemented in heritage documentation. Furthermore, considering the 
variability of distance to object plane of long-focus lenses one can draw conclusions that not only calibration for lenses focused at average distances from objects can be used but also calibrations made for other specific focus distances - applied in other applications. It will obviously depend on the type of camera and the stability of internal orientation parameters. It is possible that a set of calibrations for one lens can cover all lens positions for creating orthophotoplans of flat and quasi-flat surfaces, but it would require further study.

\section{Acknowledgements}

The author wishes to acknowledge the contribution of Dr. Eng. A. Boroń to various discussions during the preparation of this paper.

\section{References}

[1] Al Ajlouni S.S., Fraser C.S.: Zoom-dependent calibration for consumer-grade cameras. ISPRS Commission V Symposium Image Engineering and Vision Metrology, 2006.

[2] Bräuer-Burchardt C., Heinze M., Munkelt C., Kühmstedt P., Notni G.: Distance Dependent Lens Distortion Variation in 3D Measuring Systems Using Fringe Projection. BMVC, 2006, pp. 327-336.

[3] Brown D.C.: Decentering Distortion of Lenses. Photogrammetric Engineering, no. 3, 1966, pp. 444-462.

[4] Brown D.C.: Close-range camera calibration. Photogrammetric Engineering, vol. 37(8), 1971, pp. 855-866.

[5] Clarke T.A., Wang X., Fryer J.G.: The principal point and CCD cameras. The Photogrammetric Record, vol. 16, issue 92, 1998, pp. 293-312.

[6] Fraser C.S.: Multiple focal setting self-calibration of close-range metric cameras. Photogrammetric Engineering and Remote Sensing, vol. 46 (9), 1980, pp. 1161-1171.

[7] Fraser C.S., Shortis M.R.: Variation of distortion within the photographic field. Photogrammetric Engineering and Remote Sensing, vol. 58(6), 1992, pp. 851-855.

[8] Fraser C.S., Shortis M.R.: A correction model for variation of distortion within the photographic field. SPIE 1395, 1990, pp. 244-251.

[9] Fryer J.G., Brown D.C.: Lens distortion for close-range Photogrammetry. Photogrammetric Engineering and Remote Sensing, vol. 52(1), 1986, pp. 51-58.

[10] Fryer J.G.: Distortion in zoom lenses. Australian Journal of Geodesy, Photogrammetry and Surveying, vol. 44, 1986, pp. 49-59. 
[11] Sawicki P.: Kalibracja równoczesna aparatu cyfrowego Kodak DC4800 w procesie fotogrametrycznego pomiaru punktów w bliskim zasięgu. Archiwum Fotogrametrii, Kartografii i Teledetekcji, vol. 13B, 2003, pp. 457-466.

[12] Shortis M.R., Bellman C.J., Robson S., Johnston G.J., Johnson G.W.: Stability of zoom and fixed lenses used with digital SLR cameras. Proceedings of the ISPRS Commission V Symposium of Image Engineering and Vision Metrology, Dresden, Germany, 2006, pp. 285-290.

[13] Shortis M.R., Robson S., Beyer H.A.: Principal point behaviour and calibration parameter models for Kodak DCS cameras. Photogrammetric Record, vol. 16, 1998, pp. 165-186.

[14] Tayman W.P., Ziemann H.: Photogrammetric camera calibration. Photogrammetria, vol. 39(2), 1984, pp. 31-53. 